\title{
nature
}

cell biology

\section{Spotlight on Metabolism}

\section{In this issue, we present the first of a series of Reviews discussing different aspects of cell metabolism.}

Metabolism has a central role in cellular and organismal growth, survival and development. Imbalances in metabolic pathways have also been linked to diseases such as cancer and obesity. The importance of metabolism in human health and disease is underscored by the extensive research efforts focused on understanding the physiological and pathophysiological natures of cellular metabolic networks.

Throughout 2015, Nature Cell Biology will present a series of Reviews that highlight emerging themes in this diverse, exciting field. We begin the series this month with a Review by Riera and Dillin that discusses how nutrient availability affects longevity, with a focus on how alternations in mitochondrial function could underpin metabolic disorders. Next month will bring a Review by Boroughs and DeBerardinis that provides insight into the connection between metabolic changes and cancer cell growth. This Review will also highlight mechanisms of 'metabolic flexibility' that enable cancer cells to thrive in nutrient-poor conditions.

Reviews appearing in future issues of the journal will likewise address fundamental subjects in metabolism. These Reviews will provide insight into the relationship between white adipose tissue and thermogenic brown adipose tissue, investigate how stress stimuli alter metabolic pathways, and explore how lysosomes - the central degradative organelle in mammals - sense and effect changes in nutrient status and metabolic pathways. Together, the Reviews in this series will turn a spotlight on new and emerging areas in this broad field.

Later this year, the Reviews will be hosted on a dedicated page of the Nature Cell Biology website, and will be accompanied by an online library presenting specially selected research papers and reviews from Nature Cell Biology and other Nature journals. We thank our authors and referees for their contributions, and hope you share our excitement and enthusiasm for this series on metabolism.

\section{Introducing double-blind peer review}

\section{Nature and its sister journals start offering anonymity to authors during the peer-review process.}

Starting in March, Nature and the monthly Nature research journals will experiment with an alternative to their time-tested method of traditional single-blind peer review, in which reviewers are anonymous but know the authors' identity. Authors will be able to choose double-blind peer review, in which both authors and reviewers are unknown to each other.

Double-blind and open peer review are often proposed as alternatives to the conventional single-blind process, with different strengths and limitations. Proponents of open peer review, where both authors and reviewers are unveiled to each other, see its transparency as a way to encourage more civil and thoughtful reviewer comments - although others are concerned that it promotes a less critical attitude. Advocates of double-blind peer review suggest that it eliminates ad hominem biases, such as those based on gender, seniority, reputation and affiliation ${ }^{1}$. How effectively either method can meet these aspirations while maintaining the necessary level of criticism remains a matter of debate.

Nature experimented with open peer review in 2006, but at the time, despite expressed interest, the uptake from both authors and reviewers was low and the open reviews were not technically substantive. Views about open peer review are probably still evolving, as several journals continue to experiment with variations on this practice. Opinions about double-blind review, however, are remarkably consistent.

In one of the largest studies on peer review - a 2009 international and cross-disciplinary survey of more than 4,000 researchers ${ }^{1}-76 \%$ of respondents indicated that double blind was an effective peer-review system. (By comparison, open and single-blind peer review were considered effective by $20 \%$ and $45 \%$ of respondents, respectively.) More recently, our own reader survey confirmed the desire to have double-blind peer review as an option. Importantly, this sentiment is widely echoed in conversations with young scientists worldwide. These conversations illustrate a widespread perception that biases based on authorship affect the traditional single-blind peer review, and they have contributed greatly to us reconsidering this proposition.

Nature journals editors have traditionally not embraced doubleblind peer review, for several reasons including scepticism of its efficacy, concern about the potential difficulty of recruiting referees, and the view that it is the editor's responsibility to mitigate biases addressed by doubleblind review. All editors take, and will continue to take, this responsibility seriously by maintaining awareness of any potential predispositions when selecting reviewers and considering their comments. They will also continue to honour reasonable requests to exclude particular reviewers, regardless of the chosen method of peer review. But by definition, unconscious biases may be difficult to identify and to control. Several studies have detected involuntary biases, notably on the basis of gender, in other areas of the scientific enterprise, such as the hiring of laboratory staff, citation habits and speaker lineups at conferences. It is therefore difficult to guarantee a bias-free process.

Since June 2013, Nature Geoscience and Nature Climate Change have allowed authors to choose between double-blind and singleblind peer review at submission ${ }^{2}$. The reactions to the trial among surveyed authors have been sufficiently positive that Nature and the Nature monthly journals have decided to join the experiment. (Nature Communications will join at a later date.)

The responsibility for rendering the manuscript anonymous falls to the authors. Clearly, in some situations, maintaining anonymity will be impossible because of awareness of their work in the specialist community or because researchers wish to release data early and to discuss their work with their peers prior to publication, via conferences or preprint servers. Therefore, the double-blind process is optional on all titles. Some will choose it to assuage concerns about biases, others purely by principle.

We will keep this initiative under review, and we welcome comments from authors and reviewers.

1. Mulligan, A., Hall, H. \& Raphael, E. J. Am. Soc. Inf. Sci. Technol. 64, 132-161 (2013). 2. Brown, A. Nat. Nanotech. 9, 871-872 (2014). 